\title{
Erratum to: Associations between DSM-IV mental disorders and diabetes mellitus: a role for impulse control disorders and depression
}

\author{
Peter de Jonge • Jordi Alonso • Dan J. Stein • Andrzej Kiejna • Sergio Aguilar-Gaxiola • \\ Maria Carmen Viana - Zhaorui Liu • Siobhan O'Neill • Ronny Bruffaerts • \\ Jose Miguel Caldas-de-Almeida • Jean-Pierre Lepine • Herbert Matschinger • \\ Daphna Levinson • Giovanni de Girolamo • Akira Fukao • Brendan Bunting • \\ Josep Maria Haro • Jose A. Posada-Villa • Ali Obaid Al-Hamzawi • \\ Maria Elena Medina-Mora • Marina Piazza • Chiyi Hu • Carmen Sasu • \\ Carmen C. W. Lim • Ronald C. Kessler • Kate M. Scott
}

Published online: 28 March 2014

(C) Springer-Verlag Berlin Heidelberg 2014

\section{Erratum to: Diabetologia}

DOI 10.1007/s00125-013-3157-9

Unfortunately, the affiliations for Dr Jordi Alonso and Dr Giovanni de Girolamo were incorrect in the original version of the paper. The correct affiliations are given below.
The online version of the original article can be found at http://dx.doi.org/ 10.1007/s00125-013-3157-9.

\section{P. de Jonge $(\square)$}

Interdisciplinary Center Psychopathology and Emotion Regulation,

University Medical Center Groningen, PO 9700 MB, Groningen,

The Netherlands

e-mail: peter.de.jonge@umcg.nl

\section{J. Alonso}

Health Services Research Unit, IMIM-Institut Hospital del Mar

d'Investigacions Mèdiques, Barcelona, Spain

\section{J. Alonso}

CIBER en Epidemiología y Salud Pública (CIBERESP), Spain

URL: www.ciberesp.es

\section{J. Alonso}

Department of Experimental and Health Sciences (CEXS),

Universitat Pompeu Fabra (UPF), Barcelona, Spain
D. J. Stein

Department of Psychiatry and Mental Health, University of Cape Town, Cape Town, South Africa

A. Kiejna

Department of Psychiatry, Wroclaw Medical University,

Wroclaw, Poland

S. Aguilar-Gaxiola

Center for Reducing Health Disparities, School of Medicine,

Sacramento, CA, USA

M. C. Viana

Department of Social Medicine, Federal University of Espírito Santo (UFES), Vitória, Espírito Santo, Brazil

Z. Liu

Institute of Mental Health, Peking University, Beijing, China 
S. O’Neill

School of Psychology, University of Ulster, Londonderry, UK

R. Bruffaerts

University Hospital Gasthuisberg, Leuven, Belgium

J. M. Caldas-de-Almeida

Chronic Diseases Research Center (CEDOC) and Department of

Mental Health, Faculdade de Ciências Médicas, Universidade Nova

de Lisboa, Lisbon, Portugal

J.-P. Lepine

Hôpital Lariboisiére Fernand Widal, Paris, France

H. Matschinger

Clinic of Psychiatry, University of Leipzig, Leipzig, Germany

D. Levinson

Mental Health Services, Ministry of Health, Jerusalem, Israel

G. de Girolamo

IRCCS Centro S. Giovanni di Dio, Fatebenefratelli, Brescia, Italy

\section{A. Fukao}

Department of Public Health, Yamagata University School of

Medicine, Yamagata, Japan

B. Bunting

Psychology Research Institute, University of Ulster,

Londonderry, UK

J. M. Haro

Parc Sanitari Sant Joan de Déu, CIBERSAM,

University of Barcelona, Barcelona, Spain
J. A. Posada-Villa

Instituto Colombiano del Sistema Nervioso,

Bogota D.C., Colombia

A. O. Al-Hamzawi

Al-Qadisiya University, College of Medicine,

Diwania Governorate, Iraq

M. E. Medina-Mora

National Institute of Psychiatry, Calzada Mexico Xochimilco, Mexico City, Mexico

M. Piazza

Facultad de Salud Publica y Administración (FASPA),

Universidad Peruana Cayetano Heredia, Lima, Peru

C. $\mathrm{Hu}$

Shenzhen Institute of Mental Health and Shenzhen Kangning Hospital, Shenzhen, Guangdong Province,

People's Republic of China

\section{Sasu}

National School of Public Health, Management and Professional Development, Bucharest, Romania

\section{C. W. Lim • K. M. Scott}

Department of Psychological Medicine, University of Otago,

Dunedin, New Zealand

R. C. Kessler

Department of Healthcare Policy, Harvard Medical School, Boston, MA, USA 